\title{
Spectrum of Imaging Findings by Dynamic MRI Post Hepatic Intervention by Loco-Regional Therapies for Hepatocellular Carcinoma
}

\author{
HEND M. ABDELHAFEEZ, M.Sc.*; MOHAMED TAHA ABDELHAQ, M.D.**; \\ ASHRAF M. ENITE, M.D.** and WESSAM MOSTAFA, M.D.* \\ The Department of Radiodiagnosis, Faculty of Medicine, Al-Azhar University* and Ministry of Health**
}

\begin{abstract}
Background: Liver cirrhosis and Hepatocellular Carcinoma (HCC) constitute one of the major causes of morbidity, mortality, and high health care costs worldwide. Multiple treatment options are available for HCC depending on the clinical status of the patient, size and location of the tumor, and available techniques and expertise. Locoregional treatment options are multiple. The most challenging part is how to assess the treatment response by different imaging modalities, but our scope will be assessing the response to locoregional therapy for HCC by MRI.
\end{abstract}

Aim of Study: To study the role of dynamic contrast enhanced MRI in follow-up of after treatment by different loco regional procedures and review the spectrum of imaging findings.

Subject and Methods: This study was conducted on 45 patients with different age group 30 to 73 years with the mean age of 58 years having different site, size and shape of hepatocellular carcinoma (proved mainly by combination of elevated alpha-fetoprotein, typically neoplastic enhancement by spiral $\mathrm{CT}$ or dynamic MRI and having hepatitis $\mathrm{C}$ or/\& $\mathrm{B}$ viruses).

Results: MRI was found to be of great value in the followup of cases of HCC that underwent locoregional therapy specially in diagnosing residual or recurrent tumor tissue in the embolized lesions and in detecting new developed lesions. This technique can be implemented simply and reliably. The study found that almost all of the embolized lesions in the unresolved group of patients (residual/recurrence) showed enhancing areas within in the arterial phase, the study also found that $68.9 \%$ of the embolized lesions in the resolved group of patients (complete tumor ablation) were not enhanced at all while $24.4 \%$ of them showed delayed marginal enhancement.

Conclusion: MRI is a powerful tool in detection of tumor viability and complications after locoregional treatment of hepatocellular carcinoma.

Correspondence to: Dr. Hend M. Abdelhafeez, The Department of Radiodiagnosis, Faculty of Medicine, Al-Azhar University
Key Words: Magnetic resonance imaging - Hepatocellular carcinoma - Locoregional treatment - Treatment response of $\mathrm{HCC}$.

\section{Introduction}

HEPATOCELLULAR Carcinoma (HCC) is a primary malignancy of the liver and occurs predominantly in patients with underlying chronic liver disease and cirrhosis. The cell(s) of origin are believed to be the hepatic stem cells, although this remains the subject of investigation, Tumors progress with local expansion, intrahepatic spread, and distant metastases [1].

Management of HCC patients depends on the stage of their tumor. Liver resection remains the first choice for very early-stage HCC, but it is being challenged by local ablative therapy. For early-stage HCC that meet the Milan criteria, liver transplantation still offers a better outcome; however, local ablative therapy can be a substitute when transplantation is not feasible. Local ablation is also used as a bridging therapy toward liver transplantation, Transarterial therapy is considered suitable for intermediate-stage HCC. Because of the complexity of HCC, therapeutic approaches must be adapted according to the characteristics of each individual patient [2]

Magnetic Resonance Imaging (MRI) of the liver is slowly transitioning from a problem solving imaging modality to a first line imaging modality for many diseases of the liver. Because of its higher diagnostic accuracy for the detection and characterization of HCCs, together with technical advancements ensuring superior and more reproducible image quality, MRI has gained an increasingly central role in evaluating patients with chronic liver disease. 
Many physicians, including liver specialists and radiologists, currently prefer dynamic contrastenhanced MRI to CT for the evaluation of liver diseases. Technological advancements in MRI that focus on producing high quality images and fast imaging, increasing diagnostic accuracy and developing newer function-specific contrast agents are essential in ensuring that MRI succeeds as a first line imaging modality [3]

\section{Patients and Methods}

\section{Patients:}

Study population:

Fourty five patients with hepatocellular carcinoma and underwent locoregional therapy were included in this study. The study was performed between March 2017 and May 2019.

\section{Inclusion criteria:}

Patient known to have hepatocellular carcinoma lesions underwent locoregional treatment.

\section{Exclusion criteria:}

- Contraindications to magnetic resonance imaging, e.g. claustrophobia, cardiac pacemakers.

- Other tumors other than hepatocellular carcinoma.

The patients were subjected to the following:

- Full clinical assessment including; recording of age, sex and clinical presentation.

- Laboratory investigations (liver biochemical profile, alpha fetoprotein).

- Abdominal MRI (pre-and dynamic post contrast study and diffusion weighted imaging).

\section{Methods and Material}

All patients included in this study had had Abdominal MRI (pre-and dynamic post contrast study and diffusion weighted imaging. Conventional MRI, post Gd-DTPA dynamic and diffusion MR imaging were performed. First blind characterization and detection of embolized focal lesions was performed, second the diffusion images with ADC values were reviewed. Then characterizing the enhancement pattern of the focal lesions was detected. MR imaging was performed on high field system (1.5 Tesla) magnet units (Achieva) using a phased array coil to cover the whole liver.

\section{Results}

Fourty five patients were included in this study, 31 males $(68.90 \%)$ and 14 females $(31.10 \%)$. The patients' age was ranging from 30 to 73 years with the mean age of 58.5 years, 38 patients $(84.4 \%)$ were above or equal 50 years while only 7 patients $(14.6 \%)$ were below 50 years old.

There were 26 patients $(57.8 \%)$ with a single embolized lesion and 19 patients $(42.4 \%)$ with multiple embolized lesions Fig. (1), 75.6\% of the embolized lesions were below $3 \mathrm{~cm}$, while $\%$ were above or equal $3 \mathrm{~cm}$ Fig. (2).

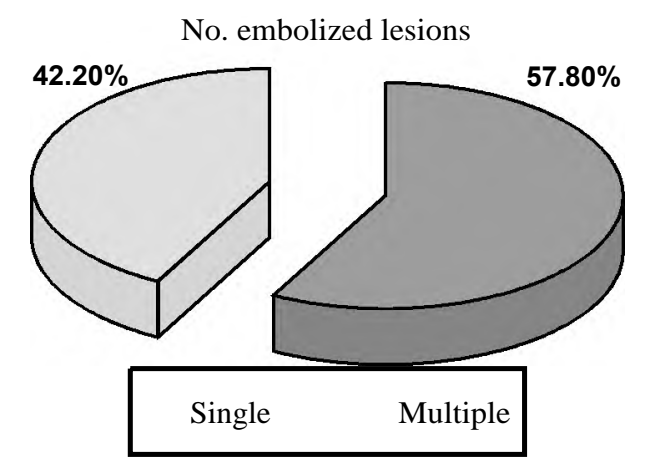

Fig. (1): Number of embolized lesions among patients.

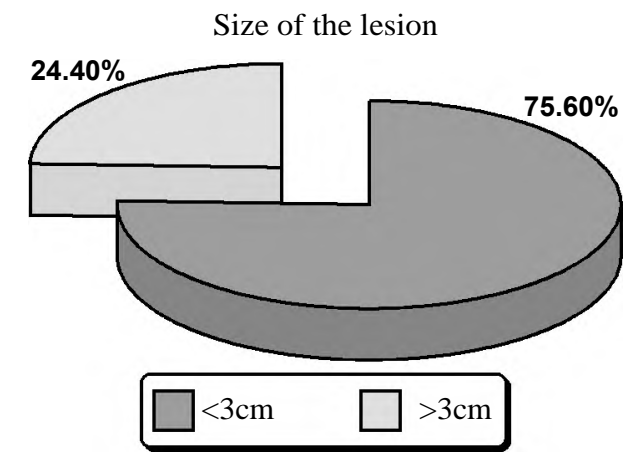

Fig. (2): Size of embolized lesions.

$31.1 \%$ of the embolized lesions showed enhancing areas within in the arterial phase (residual/ recurrence), $68.9 \%$ were not enhanced while $24.4 \%$ showed delayed marginal enhancement Fig. (3).

Recurrence/R enhancement

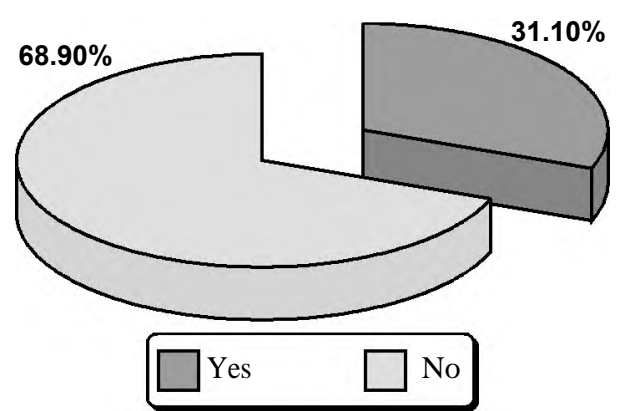

Fig. (3): Pattern of enhancement of the embolized lesions.

MRI reading reveals that $35.6 \%$ of the embolized lesions were unresolved lesions (residual/ recurrence) while $64.4 \%$ were resolved (well embolized) Fig. (4). 
MRI confirmed no residual activity

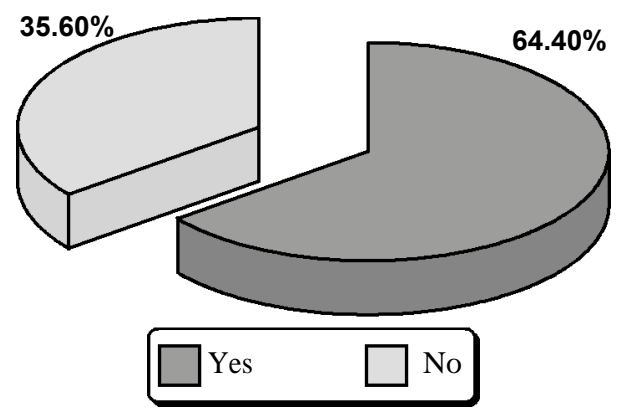

Fig. (4): Percentage of resolved and unresolved lesions.

Apart from the embolized lesions, 28.9\% of cases (13 patients) showed newly developed lesions while $71.1 \%$ (32 patients) didn't Fig. (5).

MRI detection of newly developed lesion

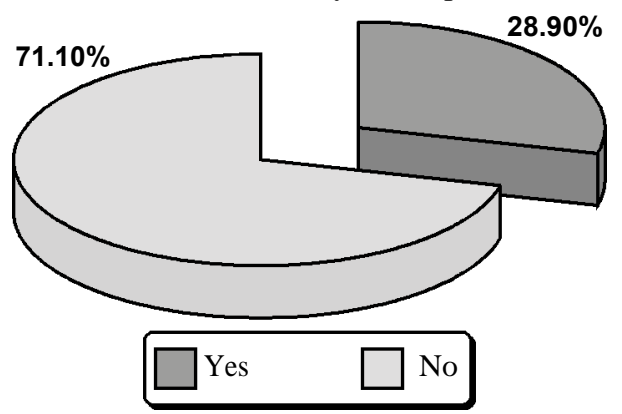

Fig. (5): Newly developed lesions.

In 32 cases $(93.3 \%)$ the portal vein was patent while it was thrombosed in 3 cases $(6.7 \%)$.
$11.1 \%$ of cases showed presence of ascites, while $88.9 \%$ of the patients didn't.

The probability of presence of residual or recurrent lesions after locoregional treatments increases with the increase in the size of the embolized lesions, as shown in the graph below Fig. (6).

The probability of presence of residual or recurrent lesions after locoregional treatments is higher in cases with child pugh grade B than in grade $\mathrm{A}$. We had only 1 case that was classified as grade $\mathrm{C}$ and it showed no recurrence/residue, as shown in the table below Fig. (7).

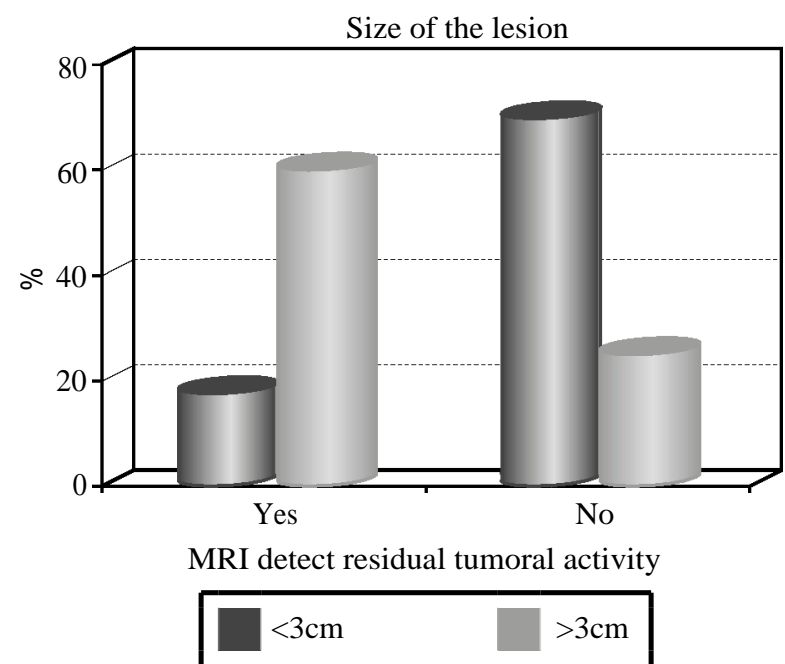

Fig. (6): Relation between the size of the embolized lesions and the probability of residual or recurrent lesions (unresolving).

\begin{tabular}{|c|c|c|c|c|c|c|c|c|c|c|c|}
\hline & \multicolumn{10}{|c|}{ Recurrence/R enhancement } & \multirow{3}{*}{$\begin{array}{c}p- \\
\text { value }\end{array}$} \\
\hline & \multicolumn{5}{|c|}{ Yes } & \multicolumn{5}{|c|}{ No } & \\
\hline & Mean & $\mathrm{SD}$ & Median & Minimum & Maximum & Mean & SD & Median & Minimum & Maximum & \\
\hline $\begin{array}{l}\text { Child pugh } \\
\text { grade } \\
\text { (points) }\end{array}$ & 6.57 & 1.02 & 7.00 & 5.00 & 9.00 & 6.81 & 1.30 & 7.00 & 5.00 & 10.00 & 0.557 \\
\hline
\end{tabular}

\begin{tabular}{|c|c|c|c|c|c|c|}
\hline & & \multicolumn{4}{|c|}{ Recurrence/R enhancement } & \multirow{3}{*}{$\begin{array}{c}p- \\
\text { value }\end{array}$} \\
\hline & & \multicolumn{2}{|c|}{ Yes } & \multicolumn{2}{|c|}{ No } & \\
\hline & & Count & $\%$ & Count & $\%$ & \\
\hline \multirow{3}{*}{$\begin{array}{l}\text { Child } \\
\text { pugh }\end{array}$} & A & 6 & 42.9 & 11 & 35.5 & \multirow[t]{3}{*}{0.826} \\
\hline & $\mathrm{B}$ & 8 & 57.1 & 19 & 61.3 & \\
\hline & $\mathrm{C}$ & 0 & 0.0 & 1 & 3.2 & \\
\hline
\end{tabular}

Fig. (7): Relation between the child pugh grading and the probability of residual or recurrent lesions (unresolving).

\section{Discussion}

The liver is an organ in which various benign or malignant primary or secondary masses can be detected. Focal masses are diagnosed using ultra- sonography and/or computed tomography. Nowadays, Magnetic Resonance Imaging (MRI) plays a key role in management of liver lesions, using a radiation-free technique and a safe contrast agent profile. MRI plays a key role in the non-invasive 
correct characterization of hepatic focal lesions. MRI is capable of providing comprehensive and highly accurate diagnostic information, with the additional advantage of lack of harmful ionizing radiation. These properties make MRI the mainstay for the noninvasive evaluation of focal liver lesions [4].

Correct detection, classification, and characterization of hepatic focal lesions are of paramount importance as they may significantly affect the choice of therapeutic approach in many cases [5]

Hepatocellular Carcinoma (HCC) is the fifth most common tumor worldwide. Multiple treatment options are available for HCC including curative resection, liver transplantation, radiofrequency ablation, trans-arterial chemoembolization, radioembolization and systemic targeted agent like sorafenib. The treatment of HCC depends on the tumor stage, patient performance status and liver function reserve and requires a multidisciplinary approach. In the past few years with significant advances in surgical treatments and locoregional therapies, the short-term survival of HCC has improved but the recurrent disease remains a big problem [6].

Tumor response and time to progression have been considered pivotal for surrogate assessment of treatment efficacy for patients with Hepatocellular Carcinoma (HCC). Recent advancements in Magnetic Resonance Imaging (MRI) are playing an important role in assessing the therapeutic effects of HCC treatments. According to some HCC clinical guidelines, post-therapeutic evaluation of HCC
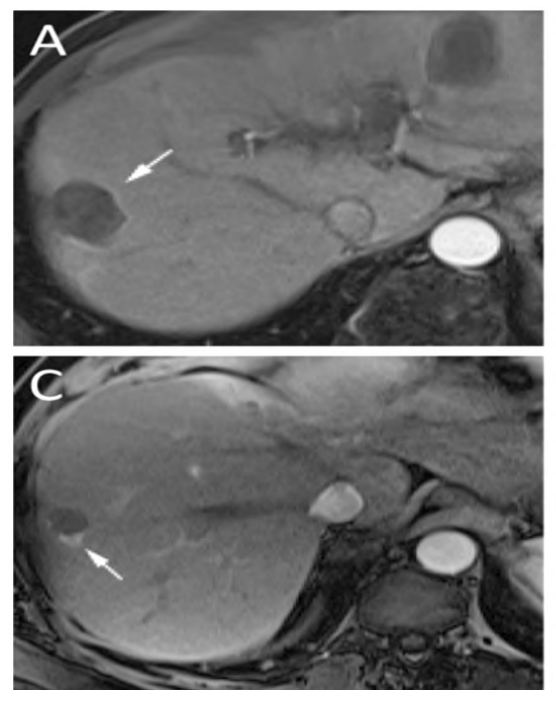

Fig (8): Post MWA treatment changes. A 62 years old patient diagnosed with HCC underwent Microwave ablation, showing no residual tumor (A and B) and recurrent nodule of disease (C and D). (A) Arterial phase imaging, 1 month after MWA demonstrates a thin (<5mm) rim (arrow) of enhancement without nodularity. (B) The thin rim of enhancement (arrow) persists on portal venous phase imaging. These findings are consistent with expected post treatment changes and not recurrence. (C) In another patient, arterial phase imaging shows a nodular area of enhancement (arrow) at the edge of the ablation zone. (D) This nodular lesion (arrow) demonstrates subtle washout on portal venous imaging.

patients is based exclusively on contrast-enhanced dynamic imaging criteria [7].

Monitoring tumor response to loco-regional therapy is an increasingly important task in oncologic imaging. Early favorable response generally indicates effectiveness of therapy, and may result in significant survival benefit. Early identification of treatment failure is also critical in patient management, since a repeat treatment cycle can be performed if liver function is maintained, before disease progression occurs [8].

Recent advances in the development of functional imaging techniques have provided the ability to detect microscopic changes in tumor microenvironment and microstructure, thus allowing the assessment of tumor response after locoregional treatment by observing alterations in tumor viability, perfusion or vascularity [7].

Dynamic contrast enhanced MR imaging can assesses the change in tumor vascularity and perfusion after TACE or local ablation therapy. Color mapping of the arterial enhancement fraction was developed in order to be used as a surrogate marker for perfusion MR [9].

DW-MRI provides unique insight into tissue cellularity, tissue organization, integrity of cells and membranes, as well as the tortuosity of the extracellular space, which can be helpful for detecting malignant diseases, and for distinguishing tumor tissues from non-tumor tissues [10].

Fig. (8) shows post microwave ablation treatment changes on MRI.
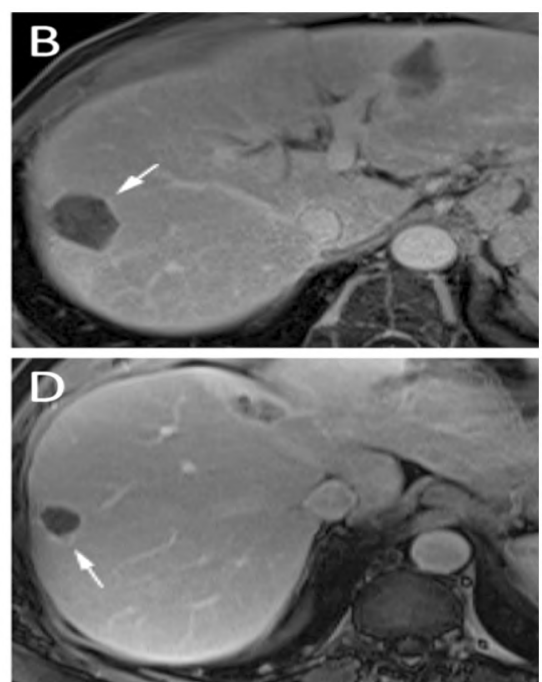

Abbreviation: MWA: Microwave Ablation. 
Also, the obvious contrast resolution of MRI especially with subtracted images helps in discriminating post-therapy changes from residual or recurrent tumor as well as detecting newly developed lesions. Moreover, MRI is superior to CT in the surveillance for patients after treatment to minimize radiation exposures and hazards of contrast agents especially if young-aged [11].

In this study, 45 patients (57.8\%) were having single embolized lesion while 10 patients $(42.2 \%)$ were having multiple embolized lesions. $24.4 \%$ of the embolized lesions were above or equal $3 \mathrm{~cm}$, while $75.6 \%$ were below $3 \mathrm{~cm}$.

The study found that $(64.4 \%)$ of the patients (resolved group) showed no MRI evidence of residual or recurrent viability at the embolized lesions while $(35.6 \%)$ of them (unresolved group) showed evidence of residual or recurrent viability.

The signal intensity of the embolized lesion in the non-enhanced $\mathrm{T} 1$ and in the $\mathrm{T} 2$ weighted images was studied and we found that $68.9 \%$ of the embolized lesions were eliciting bright $\mathrm{T} 1$ signal intensity while $31.1 \%$ were eliciting dark T 1 signal intensity, while $44 \%$ were eliciting bright $\mathrm{T} 2$ signal intensity and $56 \%$ were eliciting dark T2 signal intensity.

De santis et al., (2003) found in a corresponding similar study that $\mathrm{HCC}$ lesions eliciting bright $\mathrm{T} 1$ signal intensity post chemoembolization were $74.2 \%$ while only $33 \%$ were eliciting bright $\mathrm{T} 2$ signal intensity.

The study found that almost all of the embolized lesions in the unresolved group of patients (residual/ recurrence) showed enhancing areas within in the arterial phase, the study also found that $68.9 \%$ of the embolized lesions in the resolved group of patients (complete tumor ablation) were not enhanced at all while $24.4 \%$ of them showed delayed marginal enhancement. This is more or less similar to what Özkavukcu et al., reported in 2009 where residual tumor showed homogeneous or heterogeneous rapid enhancement on the arterial phase images while the necrotic tumoral area following TACE did not show enhancement on contrastenhanced series and that was conclusive that enhancing portions of the tumor are presumed to be viable. They also found that occasionally contrastenhanced MRI cannot distinguish viable tumor cells from reactive granulation tissue. In addition, an enhancing rim that reflects either viable tumor or reactive tissue can appear on contrast-enhanced images. And because of this hyperintense ring appearing in the late phases of dynamic series, it can be difficult to detect small residual areas located in the capsule, and this explains the cases that shows embolized lesions in the resolved group of patients in this study that showed delayed marginal enhancement; hence the importance of complementing the dynamic contrast enhanced study with the arterial subtraction phases and also with the diffusion weighted sequences.

Apart from the embolized lesions, $28.9 \%$ of cases (13 patients) showed newly developed lesions while $71.1 \%$ (32 patients) didn't.

In 32 cases $(93.3 \%)$ the portal vein was patent while it was thrombosed in 3 cases $(6.7 \%)$. Jonathon et al., (2008) and Gaurav et al., (2010), both reported that the incidence of malignant portal vein thrombosis in association with $\mathrm{HCC}$ ranges from $5 \%$ to $44 \%$ in which it demonstrates the same signal intensity and contrast enhancement pattern as the primary tumor.

So far MRI was found to be of great value in the follow-up of cases of HCC that underwent chemoembolization specially in diagnosing residual or recurrent tumor tissue in the embolized lesions and in detecting new developed lesions. This technique can be implemented simply and reliably. It offers the advantages of significantly shorter acquisition times, retrospective thin-or thick-section reconstruction from the same raw data, improved three-dimensional rendering, and high-quality liver imaging with high intrinsic soft-tissue contrast. It also provides a global overview of the abdomen. Its relative contraindications include renal impairment and sensitivity to IV contrast.

We are aware of the major limitations of this study in the form of:

- Relatively small number of patients, so future study with large number of patients would be recommended.

- Various time intervals between interventions and follow-up studies, which were determined by clinical practice and not the study design.

- The lack of histologic proof in most cases, but this is also related to clinical practice where histology is not always indicated.

\section{Conclusion:}

Monitoring tumor response after locoregional treatment is an increasingly important task in oncologic imaging. Early favorable response indicates effectiveness of therapy while early identification of treatment failure is also critical in patient 
management as further treatment will be mandatory before disease progression.

Recent advances in the development of functional MR imaging techniques have provided the ability to detect microscopic changes in tumor microenvironment and microstructure that allow better assessment of treatment response.

Dynamic contrast enhanced MR imaging can assesses the change in the tumor vascularity and perfusion. DW MRI in the liver is a relative new and increasingly used imaging technique. It has the advantage that it can be obtained during a single breath-hold; it provides unique information that reflects tissue cellularity and organization. The ADC maps can also provide quantitative measurements of tissue water diffusibility, which can be used not only for disease assessment, but also for the evaluation of disease response to treatment.

\section{References}

1- LUCA C., FRANCISC T., JOHN G., et al.: Hepatocellular Carcinoma, Medscape LIOR CHARACH, LIOR ZUSMANOVITCH.2 017. *Gideon Charach/Department of Internal Medicine C, Tel Aviv Sourasky Medical Center, Sackler Faculty of Medicine, Tel Aviv University, Tel Aviv, Israel E.M.J. Hepatol., 5 (1): 81-8, 2018.

2- SHIBO L. KATRIN H., et al. Nov.: Treatment of Hepatocellular Carcinoma: A Systematic Review, Liver Cancer, 1 (3-4): 144-58, 2012.

3- MIGUEL R., ANTÓNIO P. MATOS, et al.: Magnetic resonance imaging of the cirrhotic liver: Diagnosis of the hepatocellular carcinoma and evaluation of response to treatment-part 1; CBR: Radiol. Bras., 50 (1): 38-47, 2017.

4- ANTÓNIO P.: "Focal Liver Lesions: Practical Magnetic Resonance Imaging Approach." World Journal of Hepatology, Vol. 7, No. 16, p. 1987, doi: 10.4254/wjh.v7.i16 .1987., 2015.

5- HOLZAPFEL K., BRUEGEL M., EIBER M., et al.: Characterization of small $(</=10 \mathrm{~mm})$ focal liver lesions: Value of respiratory-triggered echo-planar diffusion-weighted MR imaging. European Journal of Radiology, 25: 31617, 2011.

6- RAZA, ALI: "Hepatocellular Carcinoma Review: Current Treatment, and Evidence-Based Medicine." World Journal of Gastroenterology, Vol. 20, No. 15, p. 4115, doi: 10.3748/ wjg.v20.i15.4115, 2014.

7- CROCETTI L., et al.: "Loco-Regional Treatment of HCC: Current Status." Clinical Radiology, Vol. 72, No. 8, pp. 626-35, doi: 10. 10 16/j.crad.2017.01.013, 2017.

8- KAMEL I.R. and MORGAN H.R.: MRI Appearance of Treated Liver Lesions. Proc. Intl. Soc. Mag. Reson. Med., 19: 2011.

9- KIM K.W., LEE J.M. and CHOI B.I.: Assessment of the treatment response of HCC. Abdom. Imaging, 36: 30014, 2011.

10- ABDELSAMED, AMR A., et al.: "The Role of Diffusion Weighted MRI in the Differentiation between Benign and Malignant Hepatic Focal Lesion." The Egyptian Journal of Hospital Medicine, Vol. 68, No. 2, pp. 1176-83, doi: $10.12816 / 00390462017$.

11- RASHA S. HUSSEIN, et al.: "MRI Assessment of Hepatocellular Carcinoma after Locoregional Therapy." Insights into Imaging, Vol. 10, No. 1, doi: 10.1186/s13244-0190690-1, 2019.

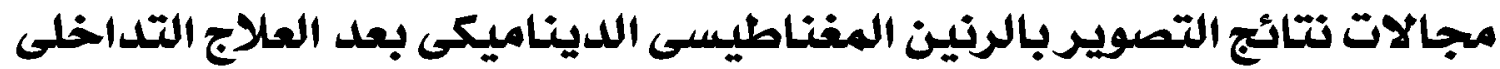 لمرضى آورام الكبد الآولية الخغبيثية الينائي}

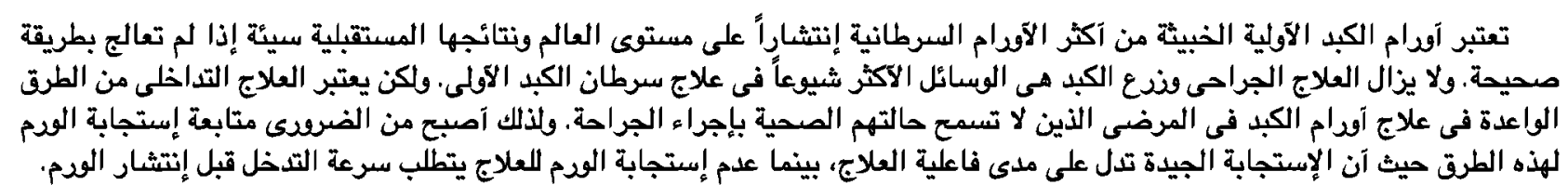

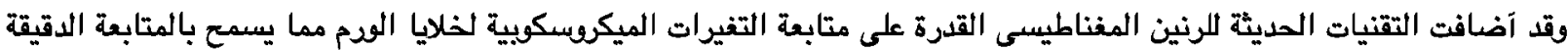

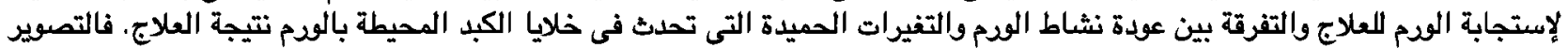

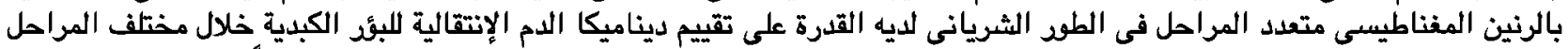

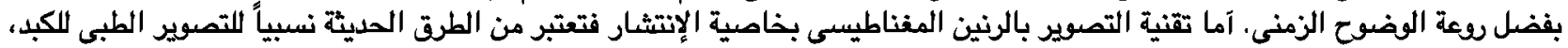

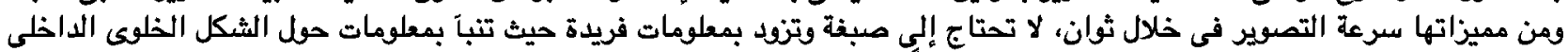

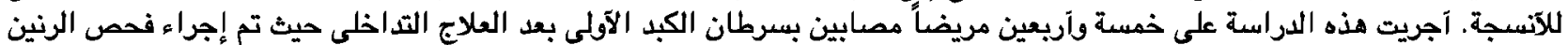

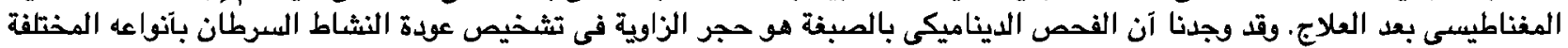

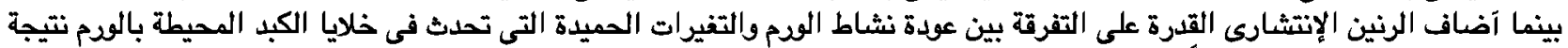

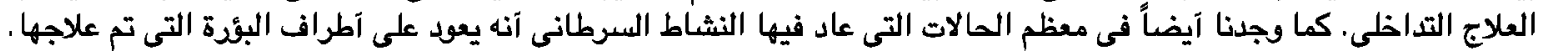

